\title{
COMPUTING NODE POLYNOMIALS FOR PLANE CURVES
}

\author{
FLORIAN BLOCK
}

\begin{abstract}
According to the Göttsche conjecture (now a theorem), the degree $N^{d, \delta}$ of the Severi variety of plane curves of degree $d$ with $\delta$ nodes is given by a polynomial in $d$, provided $d$ is large enough. These "node polynomials" $N_{\delta}(d)$ were determined by Vainsencher and Kleiman-Piene for $\delta \leq 6$ and $\delta \leq 8$, respectively. Building on ideas of Fomin and Mikhalkin, we develop an explicit algorithm for computing all node polynomials, and use it to compute $N_{\delta}(d)$ for $\delta \leq 14$. Furthermore, we improve the threshold of polynomiality and verify Göttsche's conjecture on the optimal threshold up to $\delta \leq 14$. We also determine the first nine coefficients of $N_{\delta}(d)$, for general $\delta$, settling and extending a 1994 conjecture of Di Francesco and Itzykson.
\end{abstract}

\section{Introduction and main results}

1.1. Node polynomials. Counting algebraic plane curves is a very old problem. In 1848, J. Steiner determined that the number of curves of degree $d$ with 1 node through $\frac{d(d+3)}{2}-1$ generic points in the complex projective plane $\mathbb{P}^{2}$ is $3(d-1)^{2}$. Much effort has since been put forth towards answering the following question:

How many (possibly reducible) degree d nodal curves with

$\delta$ nodes pass through $\frac{d(d+3)}{2}-\delta$ generic points in $\mathbb{P}^{2}$ ?

The answer to this question is the Severi degree $N^{d, \delta}$, the degree of the corresponding Severi variety. In 1994, Di Francesco and Itzykson [6] conjectured that $N^{d, \delta}$ is given by a polynomial in $d$ (assuming $\delta$ is fixed and $d$ is sufficiently large). It is not hard to see that, if such a polynomial exists, it has to be of degree $2 \delta$.

Recently, Fomin and Mikhalkin [7, Theorem 5.1] established the polynomiality of $N^{d, \delta}$ using tropical geometry and floor decompositions. More precisely, they showed that there exists, for every $\delta \geq 1$, a node polynomial $N_{\delta}(d)$ which satisfies $N^{d, \delta}=$ $N_{\delta}(d)$ for all $d \geq 2 \delta$. (The $\delta=0$ case is trivial as $N^{d, 0}=1$ for all $d \geq 1$.)

For $\delta=1,2,3$, the polynomiality of the Severi degrees and the formulas for $N_{\delta}(d)$ were determined in the 19th century. For $\delta=4,5,6$, this was only achieved by Vainsencher [13] in 1995. In 2001, Kleiman and Piene [9] settled the cases $\delta=7,8$. Earlier, Göttsche [8] conjectured a more detailed (still not entirely explicit) description of these polynomials for counting nodal curves on smooth projective algebraic surfaces.

1.2. Main results. In this paper we develop, building on ideas of Fomin and Mikhalkin [7], an explicit algorithm (see Algorithm 1) for computing the node polynomials $N_{\delta}(d)$ for arbitrary $\delta$. This algorithm is used to calculate $N_{\delta}(d)$ for all $\delta \leq 14$.

Received by the editors June 10, 2010. Revision received October 27, 2010.

Key words and phrases. Severi degree, Göttsche conjecture, node polynomials, floor diagram. 2010 Mathematics Subject Classification: Primary: 14N10. Secondary: 14T05, 14N35, 05A99. 
Theorem 1.1. The node polynomials $N_{\delta}(d)$, for $\delta \leq 14$, are as listed in Appendix $A$.

Di Francesco and Itzykson [6] conjectured the first seven terms of the node polynomial $N_{\delta}(d)$, for arbitrary $\delta$. We confirm and extend their assertion. The first two terms already appeared in [9].

Theorem 1.2. The first nine coefficients of $N_{\delta}(d)$ are given by

$$
\begin{aligned}
N_{\delta}(d)= & \frac{3^{\delta}}{\delta !}\left[d^{2 \delta}-2 \delta d^{2 \delta-1}-\frac{\delta(\delta-4)}{3} d^{2 \delta-2}+\frac{\delta(\delta-1)(20 \delta-13)}{6} d^{2 \delta-3}+\right. \\
& -\frac{\delta(\delta-1)\left(69 \delta^{2}-85 \delta+92\right)}{54} d^{2 \delta-4}-\frac{\delta(\delta-1)(\delta-2)\left(702 \delta^{2}-629 \delta-286\right)}{270} d^{2 \delta-5}+ \\
& +\frac{\delta(\delta-1)(\delta-2)\left(6028 \delta^{3}-15476 \delta^{2}+11701 \delta+4425\right)}{3240} d^{2 \delta-6}+ \\
& +\frac{\delta(\delta-1)(\delta-2)(\delta-3)\left(13628 \delta^{3}-6089 \delta^{2}-29572 \delta-24485\right)}{11340} d^{2 \delta-7}+ \\
& \left.-\frac{\delta(\delta-1)(\delta-2)(\delta-3)\left(282855 \delta^{4}-931146 \delta^{3}+417490 \delta^{2}+425202 \delta+1141616\right)}{204120} d^{2 \delta-8}+\cdots\right] .
\end{aligned}
$$

Let $d^{*}(\delta)$ denote the polynomiality threshold for Severi degrees, i.e., the smallest positive integer $d^{*}=d^{*}(\delta)$ such that $N_{\delta}(d)=N^{d, \delta}$ for $d \geq d^{*}$. As mentioned above Fomin and Mikhalkin showed that $d^{*} \leq 2 \delta$. We improve this as follows:

Theorem 1.3. For $\delta \geq 1$, we have $d^{*}(\delta) \leq \delta$.

In other words, $N^{d, \delta}=N_{\delta}(d)$ provided $d \geq \delta \geq 1$. Göttsche [8, Conjecture 4.1] conjectured that $d^{*} \leq\left\lceil\frac{\delta}{2}\right\rceil+1$ for $\delta \geq 1$. This was verified for $\delta \leq 8$ by Kleiman and Piene [9]. By direct computation we can push it further.

Proposition 1.4. For $3 \leq \delta \leq 14$, we have $d^{*}(\delta)=\left\lceil\frac{\delta}{2}\right\rceil+1$.

That is, Göttsche's threshold is correct and sharp for $3 \leq \delta \leq 14$. For $\delta=1,2$ it is easy to see that $d^{*}(1)=1$ and $d^{*}(2)=1$.

Di Francesco and Itzykson [6] hypothesized that $d^{*}(\delta) \leq\left\lceil\frac{3}{2}+\sqrt{2 \delta+\frac{1}{4}}\right\rceil$ (which is equivalent to $\left.\delta \leq \frac{\left(d^{*}-1\right)\left(d^{*}-2\right)}{2}\right)$. However, our computations show that this fails for $\delta=13$ as $d^{*}(13)=8$.

The main techniques of this paper are combinatorial. By the celebrated Correspondence Theorem of Mikhalkin [11, Theorem 1] one can replace the algebraic curve count by an enumeration of certain tropical curves. Brugallé and Mikhalkin [3, 4] introduced some purely combinatorial gadgets, called (marked) labeled floor diagrams (see Section 2), which, if counted correctly, are equinumerous to these tropical curves. Recently, Fomin and Mikhalkin [7] enhanced Brugallé and Mikhalkin's definition and introduced a template decomposition of labeled floor diagrams which is crucial in the proofs of all results in this paper, as is the reformulation of algebraic plane curve counts in terms of labeled floor diagrams (see Theorem 2.5).

This paper is organized as follows: In Section 2 we review labeled floor diagrams, their markings, and their relationship with the enumeration of plane algebraic curves. The proofs of Theorems 1.1 and 1.2 are algorithmic in nature and involve a computer computation. We describe both algorithms in detail in Sections 3 and 5, respectively. The first algorithm computes the node polynomials $N_{\delta}(d)$ for arbitrary $\delta$, the second determines a prescribed number of leading terms of $N_{\delta}(d)$. 
The latter algorithm relies on the polynomiality of solutions of certain polynomial difference equations: This polynomiality has been verified for pertinent values of $\delta$ (see Section 5). Proposition 1.4 is proved in Section 3 by comparison of the numerical values of $N_{\delta}(d)$ and $N^{d, \delta}$ for various $d$ and $\delta$ (see Appendices A and B). Theorem 1.3 is proved in Section 4 .

1.3. Competing approaches: floor diagrams vs. Caporaso-Harris recursion. An alternative approach to computing the node polynomials $N_{\delta}(d)$ combines polynomial interpolation with the Caporaso-Harris recursion [5]. Once a polynomiality threshold $d_{0}(\delta)$ has been established (i.e., once we have proved that $N_{\delta}(d)=N^{d, \delta}$ for $d \geq d_{0}(\delta)$ ), we can use the recursion to determine a sufficient number of Severi degrees $N^{d, \delta}$ for $d \geq d_{0}(\delta)$, from which we then interpolate.

This approach was first used by Göttsche [8, Remark 4.1(1)]. He conjectured [8, Conjecture 4.1] the polynomiality threshold $d_{0}(\delta)=\left\lceil\frac{\delta}{2}\right\rceil+1$, and combined it with the "Göttsche-Yau-Zaslow formula" [8, Conjecture 2.4] (now a theorem of Tzeng [12]) to calculate the putative node polynomials $N_{\delta}(d)$ for $\delta \leq 28$. The Göttsche-YauZaslow formula is a stronger version of polynomiality that allows one to compute each next node polynomial by calculating only two additional Severi degrees $N^{d_{0}(\delta), \delta}$ and $N^{d_{0}(\delta)+1, \delta}$, which is done via the Caporaso-Harris formula. Since Göttsche's threshold $d_{0}(\delta)=\left\lceil\frac{\delta}{2}\right\rceil+1$ remains open as of this writing, the algorithm he used to compute the node polynomials is still awaiting a rigorous justification.

The first polynomiality threshold $d_{0}(\delta)=2 \delta$ was established by Fomin and Mikhalkin [7, Theorem 5.1]. Using this result, one can compute $N_{\delta}(d)$ for $\delta \leq 9$ but hardly any further ${ }^{1}$. With the threshold $d_{0}(\delta)=\delta$ established in Theorem 1.3, it should be possible to compute $N_{\delta}(d)$ for $\delta \leq 16$ or perhaps $\delta \leq 17$.

By contrast, our Algorithm 1 does not involve interpolation nor does it require an a priori knowledge of a polynomiality threshold. Our computations verify the results of Göttsche's calculations for $\delta \leq 14$. In our implementations, Algorithm 1 is roughly as efficient as the interpolation method discussed above. (We repeat that the latter method depends on the threshold obtained using floor diagrams.)

1.4. Gromov-Witten invariants. The Gromov-Witten invariant $N_{d, g}$ enumerates irreducible plane curves of degree $d$ and genus $g$ through $3 d+g-1$ generic points in $\mathbb{P}^{2}$. Algorithm 1 (with minor adjustments, cf. Theorem 2.5(2)) can be used to directly compute $N_{d, g}$, without resorting to a recursion involving relative GromovWitten invariants à la Caporaso-Harris [5].

1.5. Follow-up work. By extending ideas of Fomin and Mikhalkin [7] and of the present paper, we can obtain polynomiality results for relative Severi degrees, the degrees of generalized Severi varieties (see [5, 14]). This is discussed in the separate paper [1]; see Remark 3.9.

Gathmann, Markwig and the author [2] defined Psi-floor diagrams which enumerate plane curves satisfying point and tangency conditions as well as conditions given by Psi-classes. We prove a Caporaso-Harris type recursion for Psi-floor diagrams, and show that relative descendant Gromov-Witten invariants equal their tropical counterparts.

\footnotetext{
${ }^{1}$ We used an efficient C implementation of the Caporaso-Harris recursion by A. Gathmann.
} 


\section{Labeled floor diagrams}

Labeled floor diagrams are combinatorial gadgets which, if counted correctly, enumerate plane curves with certain prescribed properties. Brugallé and Mikhalkin introduced them in [3] (in slightly different notation) and studied them further in [4]. To keep this paper self-contained and to fix notation we review them and their markings following [7] where the framework that best suits our purposes was introduced.

Definition 2.1. A labeled floor diagram $\mathcal{D}$ on a vertex set $\{1, \ldots, d\}$ is a directed graph (possibly with multiple edges) with positive integer edge weights $w(e)$ satisfying:

(1) The edge directions respect the order of the vertices, i.e., for each edge $i \rightarrow j$ of $\mathcal{D}$ we have $i<j$.

(2) (Divergence condition) For each vertex $j$ of $\mathcal{D}$, we have

$$
\operatorname{div}(j) \stackrel{\text { def }}{=} \sum_{\substack{\text { edges } e \\ j \stackrel{e}{\rightarrow} k}} w(e)-\sum_{\substack{\text { edges } e \\ i \stackrel{e}{\rightarrow} j}} w(e) \leq 1 .
$$

This means that at every vertex of $\mathcal{D}$ the total weight of the outgoing edges is larger by at most 1 than the total weight of the incoming edges.

The degree of a labeled floor diagram $\mathcal{D}$ is the number of its vertices. It is connected if its underlying graph is. Note that in [7] labeled floor diagrams are required to be connected. If $\mathcal{D}$ is connected its genus is the genus of the underlying graph (or the first Betti number of the underlying topological space). The cogenus of a connected labeled floor diagram $\mathcal{D}$ of degree $d$ and genus $g$ is given by $\delta(\mathcal{D})=\frac{(d-1)(d-2)}{2}-g$. If $\mathcal{D}$ is not connected, let $d_{1}, d_{2}, \ldots$ and $\delta_{1}, \delta_{2}, \ldots$ be the degrees and cogenera, respectively, of its connected components. Then the cogenus of $\mathcal{D}$ is $\sum_{j} \delta_{j}+\sum_{j<j^{\prime}} d_{j} d_{j^{\prime}}$. Via the correspondence between algebraic curves and labeled floor diagrams [7, Theorem $3.9]$ these notions correspond literally to the respective analogues for algebraic curves. Connectedness corresponds to irreducibility. Lastly, a labeled floor diagram $\mathcal{D}$ has multiplicity $^{2}$

$$
\mu(\mathcal{D})=\prod_{\text {edges } e} w(e)^{2} .
$$

We draw labeled floor diagrams using the convention that vertices in increasing order are arranged left to right. Edge weights of 1 are omitted.

Example 2.2. An example of a labeled floor diagram of degree $d=4$, genus $g=1$, cogenus $\delta=2$, divergences $1,1,0,-2$, and multiplicity $\mu=4$ is drawn below.

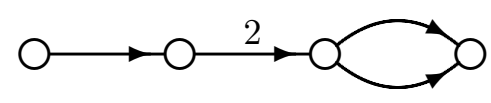

To enumerate algebraic curves via labeled floor diagrams we need the notion of markings of such diagrams.

\footnotetext{
${ }^{2}$ If floor diagrams are viewed as floor contractions of tropical plane curves this corresponds to the notion of multiplicity of tropical plane curves.
} 
Definition 2.3. A marking of a labeled floor diagram $\mathcal{D}$ is defined by the following three-step process which we illustrate in the case of Example 2.2.

Step 1. For each vertex $j$ of $\mathcal{D}$ create $1-\operatorname{div}(j)$ many new vertices and connect them to $j$ with new edges directed away from $j$.

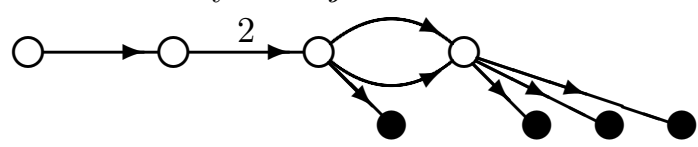

Step 2. Subdivide each edge of the original labeled floor diagram $\mathcal{D}$ into two directed edges by introducing a new vertex for each edge. The new edges inherit their weights and orientations. Call the resulting graph $\tilde{\mathcal{D}}$.

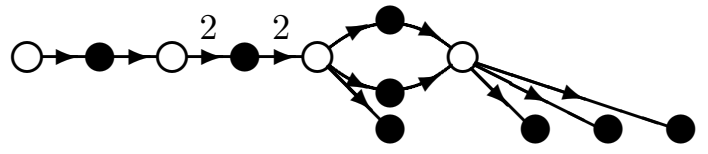

Step 3. Linearly order the vertices of $\tilde{\mathcal{D}}$ extending the order of the vertices of the original labeled floor diagram $\mathcal{D}$ such that, as before, each edge is directed from a smaller vertex to a larger vertex.

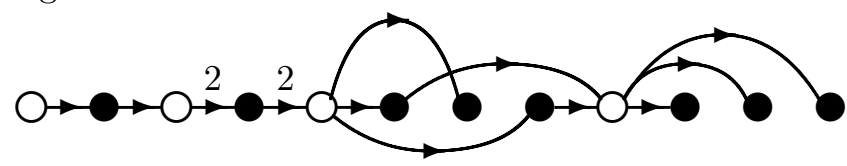

The extended graph $\tilde{\mathcal{D}}$ together with the linear order on its vertices is called a marked floor diagram, or a marking of the original labeled floor diagram $\mathcal{D}$.

We want to count marked floor diagrams up to equivalence. Two markings $\tilde{\mathcal{D}}_{1}$, $\tilde{\mathcal{D}}_{2}$ of a labeled floor diagram $\mathcal{D}$ are equivalent if there exists an automorphism of weighted graphs which preserves the vertices of $\mathcal{D}$ and maps $\tilde{\mathcal{D}}_{1}$ to $\tilde{\mathcal{D}}_{2}$. The number of markings $\nu(\mathcal{D})$ is the number of marked floor diagrams $\tilde{\mathcal{D}}$ up to equivalence.

Example 2.4. The labeled floor diagram $\mathcal{D}$ of Example 2.2 has $\nu(\mathcal{D})=7$ markings (up to equivalence): In step 3 the extra 1-valent vertex connected to the third white vertex from the left can be inserted in three ways between the third and fourth white vertex (up to equivalence) and in four ways right of the fourth white vertex (again up to equivalence).

Now we can make precise how to compute Severi degrees $N^{d, \delta}$ and Gromov-Witten invariants $N_{d, g}$ in terms of combinatorics of labeled floor diagrams, thereby reformulating the initial question of this paper. Part 2.5 is not needed in the sequel and only included for completeness. It first appeared in [3, Theorem 1].

Theorem 2.5. ([7, Corollary 1.9, Theorem 1.6]).

(1) The Severi degree $N^{d, \delta}$, i.e., the number of possibly reducible nodal curves in $\mathbb{P}^{2}$ of degree $d$ with $\delta$ nodes through $\frac{d(d+3)}{2}-\delta$ generic points, is equal to

$$
N^{d, \delta}=\sum_{\mathcal{D}} \mu(\mathcal{D}) \nu(\mathcal{D})
$$

where $\mathcal{D}$ runs over all possibly disconnected labeled floor diagrams of degree $d$ and cogenus $\delta$. 
(2) The Gromov-Witten invariant $N_{d, g}$, i.e., the number of irreducible curves in $\mathbb{P}^{2}$ of degree $d$ and genus $g$ through $3 d+g-1$ generic points, is equal to

$$
N_{d, g}=\sum_{\mathcal{D}} \mu(\mathcal{D}) \nu(\mathcal{D})
$$

where $\mathcal{D}$ runs over all connected labeled floor diagrams of degree $d$ and genus $g$.

\section{Computing node polynomials}

In this section we give an explicit algorithm that symbolically computes the node polynomials $N_{\delta}(d)$, for given $\delta \geq 1$. (As $N^{d, 0}=1$ for $d \geq 1$, we put $N_{0}(d)=1$.) An implementation of this algorithm was used to prove Theorem 1.1 and Proposition 1.4. We mostly follow the notation in [7, Section 5]. First, we rephrase Theorem 1.1 in more compact notation. For $\delta \leq 8$ one recovers [9, Theorem 3.1]. For $\delta \leq 14$ this coincides with the conjectural formulas of [8, Remark 2.5].

Theorem 3.1. The node polynomials $N_{\delta}(d)$, for $\delta \leq 14$, are given by the generating function $\sum_{\delta \geq 0} N_{\delta}(d) x^{\delta}$ via the transformation

$$
\sum_{\delta \geq 0} N_{\delta}(d) x^{\delta}=\exp \left(\sum_{\delta \geq 1} Q_{\delta}(d) x^{\delta}\right)
$$

where

$$
\begin{aligned}
Q_{1}(d) & =3(d-1)^{2} \\
Q_{2}(d) & =\frac{-3}{2}(d-1)(14 d-25), \\
Q_{3}(d) & =\frac{1}{3}\left(690 d^{2}-2364 d+1899\right), \\
Q_{4}(d) & =\frac{1}{4}\left(-12060 d^{2}+47835 d-45207\right), \\
Q_{5}(d) & =\frac{1}{5}\left(217728 d^{2}-965646 d+1031823\right), \\
Q_{6}(d) & =\frac{1}{6}\left(-4010328 d^{2}+19451628 d-22907925\right), \\
Q_{7}(d) & =\frac{1}{7}\left(74884932 d^{2}-391230216 d+499072374\right), \\
Q_{8}(d) & =\frac{1}{8}\left(-1412380980 d^{2}+7860785643 d-10727554959\right), \\
Q_{9}(d) & =\frac{1}{9}\left(26842726680 d^{2}-157836614730 d+228307435911\right), \\
Q_{10}(d) & =\frac{1}{10}\left(-513240952752 d^{2}+3167809665372 d-4822190211285\right), \\
Q_{11}(d) & =\frac{1}{11}\left(9861407170992 d^{2}-63560584231524 d+101248067530602\right), \\
Q_{12}(d) & =\frac{1}{12}\left(-190244562607008 d^{2}+1275088266948600 d-2115732543025293\right), \\
Q_{13}(d) & =\frac{1}{13}\left(3682665360521280 d^{2}-25576895657724768 d+44039919476860362\right), \\
Q_{14}(d) & =\frac{1}{14}\left(-71494333556133600 d^{2}+513017995615177680 d-913759995239314452\right) .
\end{aligned}
$$

In particular, all $Q_{\delta}(d)$, for $1 \leq \delta \leq 14$, are quadratic in $d$.

Göttsche [8] conjectured that all $Q_{\delta}(d)$ are quadratic. This theorem proves his conjecture for $\delta \leq 14$.

The basic idea of the algorithm (see [7, Section 5]) is to decompose labeled floor diagrams into smaller building blocks. These gadgets will be crucial in the proofs of all theorems in this paper. 
Definition 3.2. A template $\Gamma$ is a directed graph (with possibly multiple edges) on vertices $\{0, \ldots, l\}$, for $l \geq 1$, and edge weights $w(e) \in \mathbb{Z}_{>0}$, satisfying:

(1) if $i \rightarrow j$ is an edge then $i<j$;

(2) every edge $i \stackrel{e}{\rightarrow} i+1$ has weight $w(e) \geq 2$ (No "short edges.");

(3) for each vertex $j, 1 \leq j \leq l-1$, there is an edge "covering" it, i.e., there exists an edge $i \rightarrow k$ with $i<j<k$.

Every template $\Gamma$ comes with some numerical data associated with it. Its length $l(\Gamma)$ is the number of vertices minus 1 . The product of squares of the edge weights is its multiplicity $\mu(\Gamma)$. Its cogenus $\delta(\Gamma)$ is

$$
\delta(\Gamma)=\sum_{i \rightarrow \mathfrak{a} j}[(j-i) w(e)-1] .
$$

For $1 \leq j \leq l(\Gamma)$ let $\varkappa_{j}=\varkappa_{j}(\Gamma)$ denote the sum of the weights of edges $i \rightarrow k$ with $i<j \leq k$ and define

$$
k_{\min }(\Gamma)=\max _{1 \leq j \leq l}\left(\varkappa_{j}-j+1\right) .
$$

This makes $k_{\min }(\Gamma)$ the smallest positive integer $k$ such that $\Gamma$ can appear in a floor diagram on $\{1,2, \ldots\}$ with left-most vertex $k$. Lastly, set

$$
\varepsilon(\Gamma)= \begin{cases}1 & \text { if all edges arriving at } l \text { have weight } 1, \\ 0 & \text { otherwise }\end{cases}
$$

Figure 1 (Figure 10 taken from [7]) lists all templates $\Gamma$ with $\delta(\Gamma) \leq 2$.

A labeled floor diagram $\mathcal{D}$ with $d$ vertices decomposes into an ordered collection $\left(\Gamma_{1}, \ldots, \Gamma_{m}\right)$ of templates as follows: First, add an additional vertex $d+1(>d)$ to $\mathcal{D}$ along with, for every vertex $j$ of $\mathcal{D}, 1-\operatorname{div}(j)$ new edges of weight 1 from $j$ to the new vertex $d+1$. The resulting floor diagram $\mathcal{D}^{\prime}$ has divergence 1 at every vertex coming

\begin{tabular}{|c|c|c|c|c|c|c|c|c|}
\hline$\Gamma$ & $\delta(\Gamma)$ & $\ell(\Gamma)$ & $\mu(\Gamma)$ & $\varepsilon(\Gamma)$ & $\varkappa(\Gamma)$ & $k_{\min }(\Gamma)$ & $P\left(\Gamma^{\circ} k\right)$ & $s(\Gamma)$ \\
\hline O & 1 & 1 & 4 & 0 & (2) & 2 & $k-1$ & 1 \\
\hline 00 & 1 & 2 & 1 & 1 & $(1,1)$ & 1 & $2 k+1$ & 0 \\
\hline O & 2 & 1 & 9 & 0 & (3) & 3 & $k-2$ & 1 \\
\hline$\frac{2}{2}$ & 2 & 1 & 16 & 0 & (4) & 4 & $\left(\begin{array}{c}k-2 \\
2\end{array}\right)$ & 2 \\
\hline$\alpha$ & 2 & 2 & 1 & 1 & $(2,2)$ & 2 & $\left(\begin{array}{c}2 k \\
2\end{array}\right)$ & 0 \\
\hline 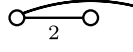 & 2 & 2 & 4 & 1 & $(3,1)$ & 3 & $2 k(k-2)$ & 1 \\
\hline 0 & 2 & 2 & 4 & 0 & $(1,3)$ & 2 & $2 k(k-1)$ & 1 \\
\hline$\sigma$ & 2 & 3 & 1 & 1 & $(1,1,1)$ & 1 & $3(k+1)$ & 0 \\
\hline$\sigma$ & 2 & 3 & 1 & 1 & $(1,2,1)$ & 1 & $k(4 k+5)$ & 0 \\
\hline
\end{tabular}
from $\mathcal{D}$. Now remove all short edges from $\mathcal{D}^{\prime}$, that is, all edges of weight 1 between consecutive vertices. The result is an ordered collection of templates $\left(\Gamma_{1}, \ldots, \Gamma_{m}\right)$,

Figure 1. The templates with $\delta(\Gamma) \leq 2$. 
listed left to right, and it is not hard to see that $\sum \delta\left(\Gamma_{i}\right)=\delta(\mathcal{D})$. This process is reversible once we record the smallest vertex $k_{i}$ of each template $\Gamma_{i}$ (see Example 3.3).

Example 3.3. An example of the decomposition of a labeled floor diagram into templates is illustrated below. Here, $k_{1}=2$ and $k_{2}=4$.

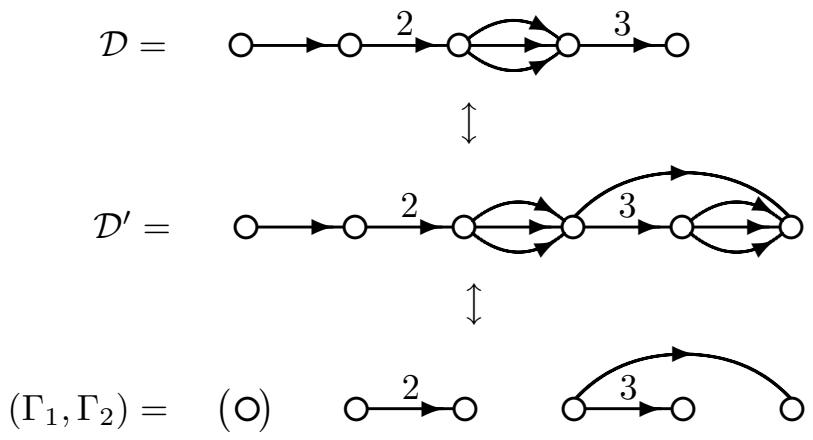

To each template $\Gamma$ we associate a polynomial that records the number of "markings of $\Gamma$ :" For $k \in \mathbb{Z}_{>0}$ let $\Gamma_{(k)}$ denote the graph obtained from $\Gamma$ by first adding $k+i-$ $1-\varkappa_{i}$ short edges connecting $i-1$ to $\mathrm{i}$, for $1 \leq i \leq l(\Gamma)$, and then subdividing each edge of the resulting graph by introducing one new vertex for each edge. By Fomin and Mikhalkin [7, Lemma 5.6] the number of linear extensions (up to equivalence) of the vertex poset of the graph $\Gamma_{(k)}$ extending the vertex order of $\Gamma$ is a polynomial in $k$, if $k \geq k_{\min }(\Gamma)$, which we denote by $P(\Gamma, k)$ (see Figure 1 ). The number of markings of a labeled floor diagram $\mathcal{D}$ decomposing into templates $\left(\Gamma_{1}, \ldots, \Gamma_{m}\right)$ is then

$$
\nu(\mathcal{D})=\prod_{i=1}^{m} P\left(\Gamma_{i}, k_{i}\right)
$$

where $k_{i}$ is the smallest vertex of $\Gamma_{i}$ in $\mathcal{D}$. The algorithm is based on

Theorem 3.4. ([7, (5.13)]). The Severi degree $N^{d, \delta}$, for $d, \delta \geq 1$, is given by the template decomposition formula

$$
\sum_{\left(\Gamma_{1}, \ldots, \Gamma_{m}\right)} \prod_{i=1}^{m} \mu\left(\Gamma_{i}\right) \sum_{k_{m}=k_{\min }\left(\Gamma_{m}\right)}^{d-l\left(\Gamma_{m}\right)+\varepsilon\left(\Gamma_{m}\right)} P\left(\Gamma_{m}, k_{m}\right) \cdots \sum_{k_{1}=k_{\min }\left(\Gamma_{1}\right)}^{k_{2}-l\left(\Gamma_{1}\right)} P\left(\Gamma_{1}, k_{1}\right),
$$

where the first sum is over all ordered collections of templates $\left(\Gamma_{1}, \ldots, \Gamma_{m}\right)$, for all $m \geq 1$, with $\sum_{i=1}^{m} \delta\left(\Gamma_{i}\right)=\delta$, and the sums indexed by $k_{i}$, for $1 \leq i<m$, are over $k_{\min }\left(\Gamma_{i}\right) \leq k_{i} \leq k_{i+1}-l\left(\Gamma_{i}\right)$,

Expression (3.1) can be evaluated symbolically, using the following two lemmata. The first is Faulhaber's formula [10] from 1631 for discrete integration of polynomials. The second treats lower limits of iterated discrete integrals and its proof is straightforward. Here $B_{j}$ denotes the $j$ th Bernoulli number with the convention that $B_{1}=+\frac{1}{2}$.

Lemma $3.5([10])$. Let $f(k)=\sum_{i=0}^{d} c_{i} k^{i}$ be a polynomial in $k$. Then, for $n \geq 0$,

$$
F(n) \stackrel{\text { def }}{=} \sum_{k=0}^{n} f(k)=\sum_{s=0}^{d} \frac{c_{s}}{s+1} \sum_{j=0}^{s}\left(\begin{array}{c}
s+1 \\
j
\end{array}\right) B_{j} n^{s+1-j} .
$$




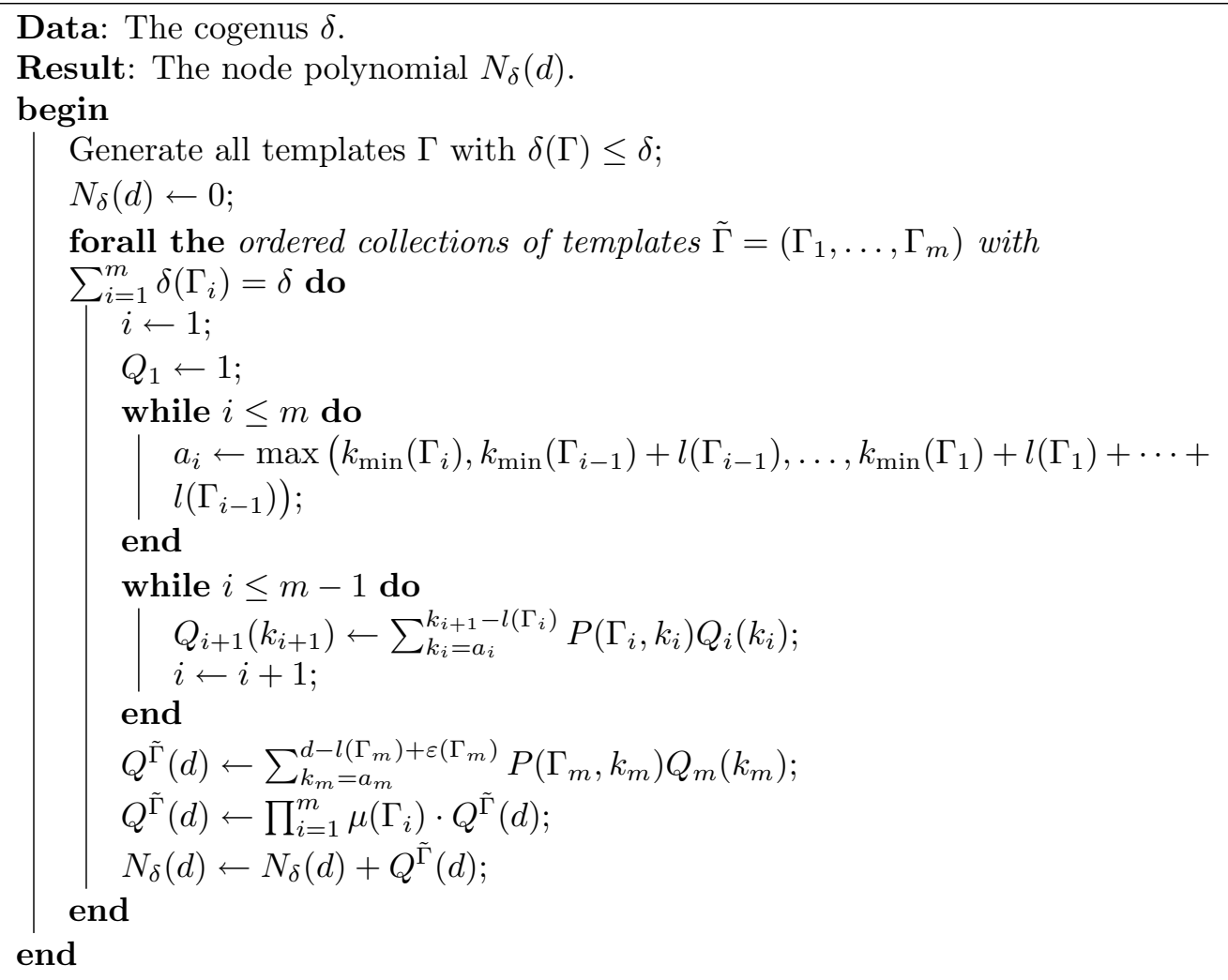

Algorithm 1: Algorithm to compute node polynomials.

In particular, $\operatorname{deg}(F)=\operatorname{deg}(f)+1$.

Lemma 3.6. Let $f\left(k_{1}\right)$ and $g\left(k_{2}\right)$ be polynomials in $k_{1}$ and $k_{2}$, respectively, and let $a_{1}, b_{1}, a_{2}, b_{2} \in \mathbb{Z}_{\geq 0}$. Furthermore, let $F\left(k_{2}\right)=\sum_{k_{1}=a_{1}}^{k_{2}-b_{1}} f\left(k_{1}\right)$ be a discrete antiderivative of $f\left(k_{1}\right)$, where $k_{2} \geq a_{1}+b_{1}$. Then, for $n \geq \max \left(a_{1}+b_{1}+b_{2}, a_{2}+b_{2}\right)$,

$$
\sum_{k_{2}=a_{2}}^{n-b_{2}} g\left(k_{2}\right) \sum_{k_{1}=a_{1}}^{k_{2}-b_{1}} f\left(k_{1}\right)=\sum_{k_{2}=\max \left(a_{1}+b_{1}, a_{2}\right)}^{n-b_{2}} g\left(k_{2}\right) F\left(k_{2}\right) .
$$

Example 3.7. An illustration of Lemma 3.6 is the following iterated discrete integral:

$$
\sum_{k_{2}=1}^{n} \sum_{k_{1}=1}^{k_{2}-2} 1=\sum_{k_{2}=1}^{n}\left\{\begin{array}{ll}
k_{2}-2 & \text { if } k_{2} \geq 2 \\
0 & \text { if } k_{2}=1
\end{array}\right\}=\sum_{k_{2}=3}^{n}\left(k_{2}-2\right)
$$

Using these results Algorithm 1 computes node polynomials $N_{\delta}(d)$ for an arbitrary number of nodes $\delta$. The first step, the template generation, is explained later in this section.

Proof of Correctness of Algorithm 1. This algorithm is a direct implementation of Theorem 3.4. The $m$-fold discrete integral is evaluated symbolically, one sum at a time, using Faulhaber's formula (Lemma 3.5). The lower limit $a_{i}$ of the $i$ th sum is given by an iterated application of Lemma 3.6. 


\begin{tabular}{r|r||r|r||r|r}
$\delta$ & \# of templates & $\delta$ & \# of templates & $\delta$ & \# of templates \\
\hline 1 & 2 & 6 & 1711 & 11 & 2233572 \\
2 & 7 & 7 & 7135 & 12 & 9423100 \\
3 & 26 & 8 & 29913 & 13 & 39769731 \\
4 & 102 & 9 & 125775 & 14 & 167885753 \\
5 & 414 & 10 & 529755 & &
\end{tabular}

Figure 2. The number of templates with cogenus $\delta \leq 14$.

As Algorithm 1 is stated its termination in reasonable time is hopeless for $\delta \geq 8$ or 9. The novelty of this section, together with an explicit formulation, is how to implement the algorithm efficiently. This is explained in Remark 3.8.

Remark 3.8. The running time of the algorithm can be improved vastly as follows: As the limits of summation in (3.1) only depend on $k_{\min }\left(\Gamma_{i}\right), l\left(\Gamma_{i}\right)$ and $\varepsilon\left(\Gamma_{m}\right)$, we can replace the template polynomials $P\left(\Gamma_{i}, k_{i}\right)$ by $\sum P\left(\Gamma_{i}, k_{i}\right)$, where the sum is over all templates $\Gamma_{i}$ with prescribed $\left(k_{\min }, l, \varepsilon\right)$. After this transformation the first sum in (3.1) is over all combinations of those tuples. This reduces the computation drastically as, for example, the 167885753 templates of cogenus 14 make up only 343 equivalence classes. Also, in (3.1) we can distribute the template multiplicities $\mu\left(\Gamma_{i}\right)$ and replace $P\left(\Gamma_{i}, k_{i}\right)$ by $\mu\left(\Gamma_{i}\right) P\left(\Gamma_{i}, k_{i}\right)$ and thereby eliminate $\prod \mu\left(\Gamma_{i}\right)$. Another speed-up is to compute all discrete integrals of monomials using Lemma 3.5 in advance.

The generation of the templates is the bottleneck of the algorithm. Their number grows rapidly with $\delta$ as can be seen from Figure 2. However, their generation can be parallelized easily (see below).

Algorithm 1 has been implemented in Maple. Computing $N_{14}(d)$ on a machine with two quad-core Intel(R) Xeon(R) CPU L5420 @ $2.50 \mathrm{GHz}, 6144 \mathrm{~KB}$ cache, and 24 GB RAM took about 70 days.

Remark 3.9. Using the combinatorial framework of floor diagrams one can show that also relative Severi degrees (i.e., the degrees of generalized Severi varieties, see [5, 14]) are polynomial and given by "relative node polynomials" [1, Theorem 1.1]. This suggests the existence of a generalization of Göttsche's Conjecture [8, Conjecture 2.1] and the Göttsche-Yau-Zaslow formula [8, Conjecture 2.1]. Thus, the combinatorics of floor diagrams lead to new conjectures although the techniques and results seem to be out of reach at this time.

Remark 3.10. We can use Algorithm 1 to compute the values of the Severi degrees $N^{d, \delta}$ for prescribed values of $d$ and $\delta$. After we specify a degree $d$ and a number of nodes $\delta$ all sums in our algorithm become finite and can be evaluated numerically. See Appendix B for all values of $N^{d, \delta}$ for $0 \leq \delta \leq 14$ and $1 \leq d \leq 13$.

Proof of Proposition 1.4. For $1 \leq \delta \leq 14$ we observe, using the data in Appendices A and $\mathrm{B}$, that $N_{\delta}(d)=N^{d, \delta}$ for all $d_{0}(\delta) \leq d<\delta$, where $d_{0}(\delta)=\left\lceil\frac{\delta}{2}\right\rceil+1$ is Göttsche's threshold. Furthermore, $N_{\delta}\left(d_{0}(\delta)-1\right) \neq N^{d_{0}(\delta)-1, \delta}$ for all $3 \leq \delta \leq 14$.

3.1. Template generation. To compute a list of all templates of a given cogenus one can proceed as follows. First, we need some terminology and notation. An edge $i \rightarrow j$ of a template is said to have length $j-i$. A template $\Gamma$ is of type $\alpha=\left(\alpha_{i j}\right)$, 


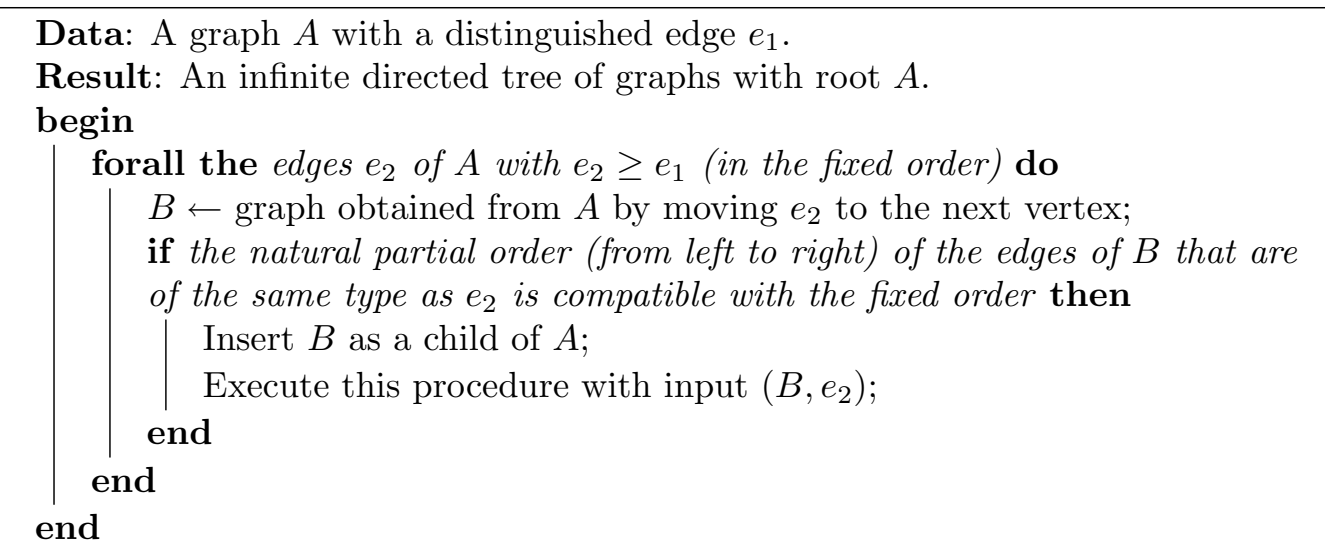

Algorithm 2: A recursion which can generates a tree containing all templates of a given type.

$i, j \in \mathbb{Z}_{>0}$, if $\Gamma$ has $\alpha_{i j}$ edges of length $i$ and weight $j$. Every type $\alpha$ satisfies, by definition of cogenus of a template,

$$
\sum_{i, j \geq 1} \alpha_{i j}(i \cdot j-1)=\delta(\Gamma) .
$$

Note that $\alpha_{11}=0$ as short edges are not allowed in templates. The number of types constituting a given cogenus $\delta$ is finite.

We can generate all templates of type $\alpha$ using a branch-and-bound algorithm which slides edges in a suitable order. Let $\Gamma_{0}$ be the unique template of type $\alpha$ with all edges emerging from vertex 0 . Fix a linear order on the set of edges of type $\alpha$. For example, if $\alpha=\left[\begin{array}{ll}0 & 1 \\ 2 & 0\end{array}\right]$, we could choose:

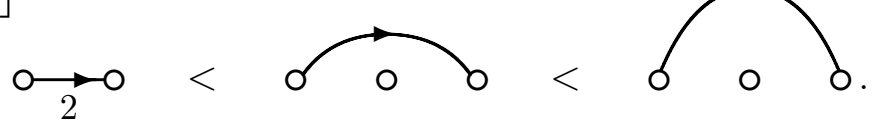

Algorithm 2 applied to the pair $\left(\Gamma_{0}, e_{0}\right)$, where $e_{0}$ is the smallest edge of $\Gamma_{0}$, creates an infinite directed tree with root $\Gamma_{0}$ all of whose vertices correspond to different graphs. Eliminate a branch if either

(1) no edge of the root of the branch starts at vertex 1 , or

(2) condition (3) in Definition 3.2 is impossible to satisfy for graphs further down the tree.

See Figure 3 for an illustration for $\alpha=\left[\begin{array}{ll}0 & 1 \\ 2 & 0\end{array}\right]$.

A complete, non-redundant list of all templates of type $\alpha$ is then given by all remaining graphs which satisfy condition (3) of Definition 3.2 as every template can be obtained in a unique way from $\Gamma_{0}$ by shifting edges in an order that is compatible with the order fixed earlier. Note that it can happen that a non-template graph precedes a template within a branch. For an example see the graph in brackets in Figure 3. Template generation for different types can be executed in parallel. The number of templates, for $\delta \leq 14$, is given in Figure 2 . 


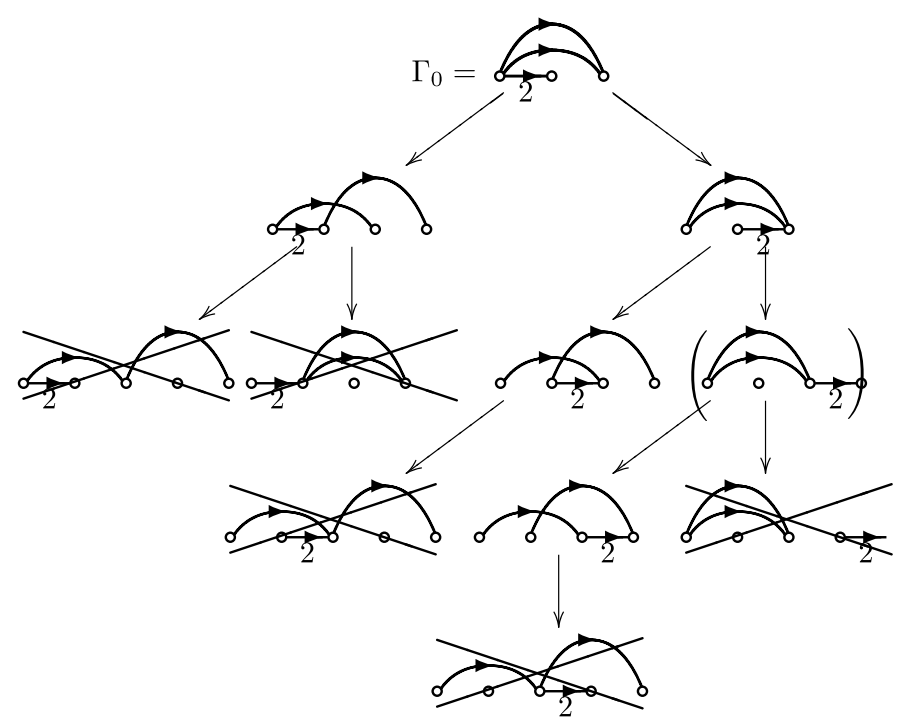

Figure 3. Branch-and-bound tree for $\alpha=\left[\begin{array}{ll}0 & 1 \\ 2 & 0\end{array}\right]$.

\section{Threshold values}

Fomin and Mikhalkin [7, Theorem 5.1] proved polynomiality of the Severi degrees $N^{d, \delta}$ in $d$, for fixed $\delta$, provided $d$ is sufficiently large. More precisely, they showed that $N_{\delta}(d)=N^{d, \delta}$ for $d \geq 2 \delta$. In this section we show that their threshold can be improved to $d \geq \delta$ (Theorem 1.3).

We need the following elementary observation about robustness of discrete antiderivatives of polynomials whose continuous counterpart is the well-known fact that $\int_{a-1}^{a-s-1} f(x) d x=0$ if $f(x)=0$ on the interval $(a-s-1, a-1)$.

Lemma 4.1. For a polynomial $f(k)$ and $a \in \mathbb{Z}_{>0}$ let $F(n)=\sum_{k=a}^{n} f(k)$ be the polynomial in $n$ uniquely determined by large enough values of $n .(F(n)$ is a polynomial by Lemma 3.5.) If we have $f(a-1)=\cdots=f(a-s)=0$ for some $0 \leq s<a$ (this condition is vacuous for $s=0)$ then it also holds that $F(a-1)=\cdots=F(a-s-1)=0$. In particular, $\sum_{k=a}^{n} f(k)$ is a polynomial in $n$, for $n \geq a-s-1$.

Even for $s=0$ the lemma is non-trivial as, in general, $F(a-2) \neq 0$.

Proof. Let $G(n)$ be the polynomial in $n$ defined via $G(n)=\sum_{k=0}^{n} f(k)$ for large $n$. Then $F(n)=G(n)-\sum_{k=0}^{a-1} f(k)$ for all $n \in \mathbb{Z}_{\geq 0}$. In particular, for any $0 \leq i \leq s$, we have $F(a-i-1)=G(a-i-1)-\sum_{k=0}^{a-1} f(k)=G(a-i-1)-\sum_{k=0}^{a-i-1} f(k)=0$.

Recall that for a template $\Gamma$, we defined $k_{\min }=k_{\min }(\Gamma)$ to be the smallest $k \geq 1$ such that $k+j-1 \geq \varkappa_{j}(\Gamma)$ for all $1 \leq j \leq l(\Gamma)$. Let $j_{0}$ be the smallest $j$ for which equality is attained (it is easy to see that equality is attained for some $j$ ). Define $s(\Gamma)$ to be the number of edges of $\Gamma$ from $j_{0}-1$ to $j_{0}$ (of any weight). See Figure 1 for some examples. The following lemma shows that the template polynomials $P(\Gamma, k)$ satisfy the condition of Lemma 4.1 . 
Lemma 4.2. With the notation from above it holds that

$$
P\left(\Gamma, k_{\min }-1\right)=P\left(\Gamma, k_{\min }-2\right)=\cdots=P\left(\Gamma, k_{\min }-s(\Gamma)\right)=0
$$

Proof. Recall from Section 3 that, for $k \geq k_{\min }(\Gamma)$, the polynomial $P(\Gamma, k)$ records the number of linear extension (up to equivalence) of some poset $\Gamma_{(k)}$ which is obtained from $\Gamma$ by first adding $k+j-1-\varkappa_{j}(\Gamma)$ "short edges" connecting $j-1$ to $j$, for $1 \leq j \leq l(\Gamma)$, and then subdividing each edge of the resulting graph by introducing a new vertex for each edge.

Using the notation from the last paragraph notice that $k_{\min }+j_{0}-1=\varkappa_{j_{0}}(\Gamma)$, and thus $\Gamma_{(k)}$ has $k-k_{\min }$ "short edges" between $j_{0}-1$ and $j_{0}$. Every linear extension of $\Gamma_{(k)}$ can be obtained by first linearly ordering the midpoints of these $k-k_{\text {min }}$ "short edges" and the midpoints of the $s(\Gamma)$ many edges of $\Gamma$ connecting $j_{0}-1$ and $j_{0}$ before completing the linear order to all vertices of $\Gamma_{(k)}$. Therefore, the polynomial $\left(k-k_{\min }+1\right) \cdots\left(k-k_{\min }+s(\Gamma)\right)$ divides $P(\Gamma, k)$.

Before we can prove Theorem 1.3 we need a last technical lemma.

Lemma 4.3. Using the notation from above we have, for each template $\Gamma$,

$$
k_{\min }(\Gamma)-s(\Gamma)+l(\Gamma)-\varepsilon(\Gamma) \leq \delta(\Gamma)+1
$$

Proof. As before, let $j_{0}$ be the smallest $j$ in $\{1, \ldots, l(\Gamma)\}$ with $k_{\min }+j-1=\varkappa_{j}(\Gamma)$. It suffices to show that $\varkappa_{j_{0}}(\Gamma)-j_{0}-s(\Gamma)+l(\Gamma)-\varepsilon(\Gamma) \leq \delta(\Gamma)$.

Let $\Gamma^{\prime}$ be the template obtained from $\Gamma$ by removing all edges $i \rightarrow k$ with either $k<j_{0}$ or $i \geq j_{0}$. It is easy to see that $l(\Gamma)-\varepsilon(\Gamma)-\left(l\left(\Gamma^{\prime}\right)-\varepsilon\left(\Gamma^{\prime}\right)\right) \leq \delta(\Gamma)-\delta\left(\Gamma^{\prime}\right)$. Thus, we can assume without loss of generality that all edges $i \rightarrow k$ of $\Gamma$ satisfy $i<j_{0} \leq k$. Therefore, as $\varkappa_{j_{0}}(\Gamma)=\sum$ edges $e$ of $\Gamma \mathrm{wt}(e)$ it suffices to show that

$$
l(\Gamma)-\varepsilon(\Gamma) \leq \sum_{\text {edges } e \text { of } \Gamma}[\operatorname{wt}(e)(\operatorname{len}(e)-1)-1]+s(\Gamma)+j_{0},
$$

where len $(e)$ is the length of an edge $e$. The contribution of the $s(\Gamma)$ edges of $\Gamma$ between $j_{0}-1$ and $j_{0}$ to the sum is $-s(\Gamma)$, thus the right-hand side of (4.1) equals

$$
\sum[\operatorname{wt}(e)(\operatorname{len}(e)-1)-1]+j_{0}
$$

with the sum now running over all edges of $\Gamma$ of length at least 2 . If there are no such edges, then $l(\Gamma)=1$ and we are done. Otherwise, if $\varepsilon(\Gamma)=1$, expression (4.2) equals $\sum(\operatorname{len}(e)-2)+j_{0}$, which is $\geq l(\Gamma)-2+j_{0}$ or $\geq l(\Gamma)-3+j_{0}$ if $j_{0} \in\{1, l(\Gamma)\}$ or $1<j_{0}<l(\Gamma)$, respectively (by considering only edges adjacent to vertices 0 and $l(\Gamma)$ of $\Gamma$ ). In either case the result follows.

If $\varepsilon(\Gamma)=0$ then expression $(4.2)$ is $\geq l(\Gamma)+\left(l(\Gamma)-3+j_{0}\right)$ or $\geq l(\Gamma)-2+j_{0}$ if $j_{0} \in\{1, l(\Gamma)\}$ or $1<j_{0}<l(\Gamma)$, respectively. This completes the proof. 
Proof of Theorem 1.3. By Lemma 3.6 and repeated application of Lemmata 4.1 and 4.2 it suffices to show that $d \geq \delta$ simultaneously implies

$$
\begin{gathered}
d \geq l\left(\Gamma_{m}\right)-\varepsilon\left(\Gamma_{m}\right)+k_{\min }\left(\Gamma_{m}\right)-s\left(\Gamma_{m}\right)-1 \\
d \geq l\left(\Gamma_{m}\right)-\varepsilon\left(\Gamma_{m}\right)+l\left(\Gamma_{m-1}\right)+k_{\min }\left(\Gamma_{m-1}\right)-s\left(\Gamma_{m-1}\right)-2 \\
\vdots \\
d \geq l\left(\Gamma_{m}\right)-\varepsilon\left(\Gamma_{m}\right)+l\left(\Gamma_{m-1}\right)+\cdots+l\left(\Gamma_{1}\right)+k_{\min }\left(\Gamma_{1}\right)-s\left(\Gamma_{1}\right)-m,
\end{gathered}
$$

for all collections of templates $\left(\Gamma_{1}, \ldots, \Gamma_{m}\right)$ with $\sum_{i=1}^{m} \delta\left(\Gamma_{i}\right)=\delta$.

The first inequality is a direct consequence of Lemma 4.3. For the other inequalities, notice that $l(\Gamma)-\varepsilon(\Gamma) \leq \delta(\Gamma)$ for all templates $\Gamma$, hence

$$
l\left(\Gamma_{m}\right)-\varepsilon\left(\Gamma_{m}\right)-1 \leq \delta\left(\Gamma_{m}\right)-1
$$

and

$$
l\left(\Gamma_{i}\right)-1 \leq \delta\left(\Gamma_{i}\right), \quad \text { for } 2 \leq i \leq m-1
$$

By Lemma 4.3 we have

$$
l\left(\Gamma_{1}\right)+k_{\min }\left(\Gamma_{1}\right)-s\left(\Gamma_{1}\right)-1 \leq \delta\left(\Gamma_{1}\right)+1
$$

as $\varepsilon\left(\Gamma_{1}\right) \leq 1$, and the right-hand-side of the last inequality of $(4.3)$ is $\leq \sum_{i=1}^{m} \delta\left(\Gamma_{i}\right)=$ $\delta \leq d$. The proof of the other inequalities is very similar.

\section{Coefficients of node polynomials}

The goal of this section is to present an algorithm for the computation of the coefficients of $N_{\delta}(d)$, for general $\delta$. The algorithm can be used to prove Theorem 1.2 and thereby confirm and extend a conjecture of Di Francesco and Itzykson in [6] where they conjectured the seven terms of $N_{\delta}(d)$ of largest degree.

Our algorithm should be able to find formulas for arbitrarily many coefficients of $N_{\delta}(d)$. We prove correctness of our algorithm in this section. The algorithm rests on the polynomiality of solutions of certain polynomial difference equations (see (5.7)).

First, we fix some notation building on terminology of Section 3. By Remark 3.8 we can replace the polynomials $P(\Gamma, k)$ in $(3.1)$ by the product $\mu(\Gamma) P(\Gamma, k)$, thereby removing the product $\prod \mu\left(\Gamma_{i}\right)$ of the template multiplicities. In this section we write $P^{*}(\Gamma, k)$ for $\mu(\Gamma) P(\Gamma, k)$. For integers $i \geq 0$ and $a \geq 0$ let $M_{i}(a)$ denote the matrix of the linear map

$$
f(k) \mapsto \sum_{\Gamma: \delta(\Gamma)=i} \sum_{k=k_{\min }(\Gamma)}^{n-l(\Gamma)} P^{*}(\Gamma, k) \cdot f(k),
$$

where $f(k)=c_{0} k^{a}+c_{1} k^{a-1}+\cdots$, a polynomial of degree $a$, is mapped to the polynomial $M_{i}(a)(f(k))=d_{0} n^{a+i+1}+d_{1} n^{a+i}+\cdots$ in $n$. (By Lemma 3.5 and the proof of Lemma 5.1 the image has degree $a+i+1$.) Hence $M_{i}(a) \mathbf{c}=\mathbf{d}$. Similarly, define $M_{i}^{\text {end }}(a)$ to be the matrix of the linear map

$$
f(k) \mapsto \sum_{\Gamma: \delta(\Gamma)=i} \sum_{k=k_{\min }(\Gamma)}^{n-l(\Gamma)+\varepsilon(\Gamma)} P^{*}(\Gamma, k) \cdot f(k)
$$


Later we will consider square sub-matrices of $M_{i}(a)$ and $M_{i}^{\text {end }}(a)$ by restriction to the first few rows and columns which will be denoted $M_{i}(a)$ and $M_{i}^{\text {end }}(a)$ as well. Note that $M_{i}(a)$ and $M_{i}^{\text {end }}(a)$ are lower triangular. For example, for $a$ large enough,

$$
M_{1}(a)=\left[\begin{array}{ccccccc}
\frac{6}{a+2} & 0 & 0 & 0 & 0 & \cdots \\
-\frac{5 a+8}{a+1} & \frac{6}{a+1} & 0 & 0 & 0 & \ldots \\
\frac{5}{2} a+3 & -\frac{5 a+3}{a} & \frac{6}{a} & 0 & 0 & \cdots \\
-\frac{1}{4}(4 a+1) a & \frac{5}{2} a+\frac{1}{2} & -\frac{5 a-2}{a-1} & \frac{6}{a-1} & 0 & \ldots \\
\frac{1}{40}\left(13 a^{2}-20 a+7\right) a & -a^{2}+\frac{7}{4} a-\frac{3}{4} & \frac{5}{2} a-2 & -\frac{5 a-7}{a-2} & \frac{6}{a-2} & \cdots \\
\vdots & \vdots & \vdots & \vdots & \vdots & \ddots
\end{array}\right] .
$$

The following observation is key to our algorithm.

Lemma 5.1. The first $a+i$ rows of $M_{i}(a)$ and $M_{i}^{\text {end }}(a)$ are independent of the lower limits of summation in (5.1) and (5.2), respectively.

Proof. It is an easy consequence of the proof of [7, Lemma 5.7] that the polynomial $P^{*}(\Gamma, k)$ associated with a template $\Gamma$ has degree $\leq \delta(\Gamma)$. Equality is attained by the template $\Gamma$ on vertices $0,1,2$ with $i$ edges connecting 0 and $2(\operatorname{so} \delta(\Gamma)=i)$. As discrete integration of a polynomial increases the degree by 1 the polynomial on the right-hand side of (5.1) has degree $1+i+a$.

The basic idea of the algorithm is that templates with higher cogenera do not contribute to higher degree terms of the node polynomial. With this in mind we define, for each finite collection $\left(\Gamma_{1}, \ldots, \Gamma_{m}\right)$ of templates, its type $\tau=\left(\tau_{2}, \tau_{3}, \ldots\right)$, where $\tau_{i}$ is the number of templates in $\left(\Gamma_{1}, \ldots, \Gamma_{m}\right)$ with cogenus equal to $i$, for $i \geq 2$. Note that we do not record the number of templates with cogenus equal to 1.

To collect the contributions of all collections of templates with a given type $\tau$, let $\tau=\left(\tau_{2}, \tau_{3}, \ldots\right.$ ) and fix $\delta \geq \sum_{j \geq 2} \tau_{j}$ (so that there exist template collections $\left(\Gamma_{1}, \ldots, \Gamma_{m}\right)$ of type $\tau$ with $\sum \delta\left(\Gamma_{j}\right)=\delta$ ). We define two (column) vectors $C_{\tau}(\delta)$ and $C_{\tau}^{\text {end }}(\delta)$ as the coefficient vectors, listed in decreasing order, of the polynomials

$$
\sum_{\left(\Gamma_{1}, \ldots, \Gamma_{m}\right)} \sum_{k_{m}=k_{\min }\left(\Gamma_{m}\right)}^{n-l\left(\Gamma_{m}\right)} P^{*}\left(\Gamma_{m}, k_{m}\right) \cdots \sum_{k_{1}=k_{\min }\left(\Gamma_{1}\right)}^{k_{2}-l\left(\Gamma_{1}\right)} P^{*}\left(\Gamma_{1}, k_{1}\right)
$$

and

$$
\sum_{\left(\Gamma_{1}, \ldots, \Gamma_{m}\right)} \sum_{k_{m}=k_{\min }\left(\Gamma_{m}\right)}^{n-l\left(\Gamma_{m}\right)+\varepsilon(\Gamma)} P^{*}\left(\Gamma_{m}, k_{m}\right) \sum_{k_{m-1}=k_{\min }\left(\Gamma_{m}-1\right)}^{k_{m}-l\left(\Gamma_{m-1}\right)} \cdots \sum_{k_{1}=k_{\min }\left(\Gamma_{1}\right)}^{k_{2}-l\left(\Gamma_{1}\right)} P^{*}\left(\Gamma_{1}, k_{1}\right)
$$

in the indeterminate $n$, where the respective first sums are over all ordered collections of templates of type $\tau$. 
It might look like $C_{\tau}(\delta)$ is a product of some matrices $M_{i}(a)$ applied to the polynomial 1. However, this is not the case. For example, note that

$$
C_{(0,0, \ldots)}(2)=\left[\begin{array}{c}
9 \\
2 \\
-34 \\
88 \\
-\frac{179}{2} \\
30 \\
0 \\
\vdots
\end{array}\right] \neq\left[\begin{array}{c}
\frac{9}{2} \\
-34 \\
88 \\
-\frac{179}{2} \\
27 \\
0 \\
\vdots
\end{array}\right]=M_{1}(2) \cdot M_{1}(0) \cdot\left[\begin{array}{c}
1 \\
0 \\
0 \\
0 \\
0 \\
0 \\
\vdots
\end{array}\right] \text {. }
$$

This is because, when iterated discrete integrals are evaluated symbolically, the lower limits of integration of the outer sums can change depending on the limits of the inner sums (cf. Lemma 3.6). This observation makes it necessary to compute initial values for recursions (described later) up to a large enough $\delta$.

Before we can state the main recursion we need two more notations. For a type $\tau=\left(\tau_{2}, \tau_{3}, \ldots\right)$ and $i \geq 2$ with $\tau_{i}>0$ define a new type $\tau \downarrow_{i}$ via $\left(\tau \downarrow_{i}\right)_{i}=\tau_{i}-1$ and $\left(\tau \downarrow_{i}\right)_{j}=\tau_{j}$ for $j \neq i$. Furthermore, let $\operatorname{def}(\tau)=\sum_{j \geq 2}(j-1) \tau_{j}$ be the defect of $\tau$. The following lemma justifies this terminology.

Lemma 5.2. Polynomials (5.3) and (5.4) are of degree $2 \delta-\operatorname{def}(\tau)$.

Proof. Let $\left(\Gamma_{1}, \ldots, \Gamma_{m}\right)$ be a collection of templates with $\sum_{i=1}^{m} \delta\left(\Gamma_{i}\right)=\delta$ and type $\tau$. Then, by applying the argument in the proof of Lemma 5.1 to each $\Gamma_{i}$, the polynomials (5.3) and (5.4) have degree $\delta+m$. The result follows as

$$
\begin{aligned}
\delta-\operatorname{def}(\tau) & =\sum_{i=1}^{m} \delta\left(\Gamma_{i}\right)-\sum_{j \geq 2}(j-1) \tau_{j} \\
& =\sum_{i=1}^{m} \delta\left(\Gamma_{i}\right)-\sum_{j \geq 2}\left[\left(\sum_{i: \delta\left(\Gamma_{i}\right)=\tau_{j}} \delta\left(\Gamma_{i}\right)\right)-\tau_{j}\right] \\
& =\#\left\{i: \delta\left(\Gamma_{i}\right)=1\right\}+\sum_{j \geq 2} \tau_{j}=m .
\end{aligned}
$$

The last lemma makes precise which collections of templates contribute to which coefficients of $N_{\delta}(d)$. Namely, the first $N$ coefficients of $N_{\delta}(d)$ of largest degree depend only on collections of templates with types $\tau$ such that $\operatorname{def}(\tau)<N$. The following recursion is the heart of the algorithm.

Proposition 5.3. For every type $\tau$ and integer $\delta$ large enough, it holds that

$$
\begin{aligned}
C_{\tau}(\delta)= & \sum_{i: \tau_{i} \neq 0} M_{i}(2 \delta-i-1-\operatorname{def}(\tau)) C_{\tau \downarrow i}(\delta-i) \\
& +M_{1}(2 \delta-2-\operatorname{def}(\tau)) C_{\tau}(\delta-1) .
\end{aligned}
$$


More precisely, if we restrict all matrices $M_{i}$ to be square of size $N-\operatorname{def}(\tau)$ and all $C_{\tau}$ to be vectors of length $N-\operatorname{def}(\tau)$, then recursion (5.5) holds for

$$
\delta \geq \max \left(\left\lceil\frac{N+1}{2}\right\rceil, \sum_{j \geq 2} j \tau_{j}\right) .
$$

Proof. The coefficient vector $C_{\tau}(\delta)$ is defined by a sum that runs over all collections of templates $\left(\Gamma_{1}, \ldots, \Gamma_{m}\right)$ of type $\tau$ (see (5.3)). Partition the set of such collections by putting $\delta\left(\Gamma_{m}\right)=1$, or $\delta\left(\Gamma_{m}\right)=2$, and so forth. This partitioning splits expression (5.3) exactly as in (5.5).

A summand can be written as a product of some matrix $M_{i}$ and some vector $C_{\tau \downarrow i}$ if $\delta$ is large enough, namely if $M_{i}$ does not depend on the lower limits in (5.3). If we can factor then the polynomials (5.3) defining $C_{\tau \downarrow i}(\delta-i)$ and $C_{\tau}(\delta-1)$ have degrees

$$
2(\delta-i)-\operatorname{def}(\tau \downarrow i)=2 \delta-2 i-\operatorname{def}(\tau)+(i-1)=2 \delta-i-1-\operatorname{def}(\tau)
$$

by Lemma 5.2 and, similarly, $2 \delta-2-\operatorname{def}(\tau)$, respectively. By Lemma 5.1 , if the matrix $M_{i}(2 \delta-i-1-\operatorname{def}(\tau))$ is of size $N-\operatorname{def}(\tau)$, then it does not depend on the lower limits if and only if $\delta \geq \frac{N+1}{2}$. In order for $C_{\tau}(\delta)$ to be defined (and the above identity to be meaningful) we need to impose $\delta \geq \sum_{j \geq 2} j \tau_{j}$.

Remark 5.4. Later, when we formulate the algorithm, we need to solve recursion (5.5) together with an initial condition in order to obtain an explicit formula for the first $N-\operatorname{def}(\tau)$ entries of $C_{\tau}(\delta)$. It suffices to take

$$
\delta_{0}(\tau) \stackrel{\text { def }}{=} \max \left(\left\lceil\frac{N-1}{2}\right\rceil, \sum_{j \geq 2} j \tau_{j}\right)
$$

as for any $\delta>\delta_{0}(\tau)$ the vector $C_{\tau}(\delta)$ of length $N-\operatorname{def}(\tau)$ can be written in terms of matrices $M_{i}$ and vectors $C_{\tau^{\prime}}\left(\delta^{\prime}\right)$ for various types $\tau^{\prime}$ and integers $\delta^{\prime}<\delta$.

We propose Algorithm 3 for the computation of the coefficients of the node polynomial $N_{\delta}(d)$. We explain how to solve recursion (5.5) below.

Proof of correctness of Algorithm 3. Proposition 5.3 guarantees that recursion (5.3) uniquely determines $C_{\tau}(\delta)$. By a similar argument as in the proof of Proposition 5.3 we see that $C_{\tau}^{\text {end }}(\delta)$ is given by the formula in Algorithm 3. By Lemma 5.2 all contributions of template collections of type $\tau$ to the node polynomial $N_{\delta}(d)$ are in degree $2 \delta-\operatorname{def}(\tau)$ or less. Hence, after shifting $C_{\tau}^{\text {end }}(\delta)$ by $\operatorname{def}(\tau)$, their sum is the coefficient vector of $N_{\delta}(d)$.

To solve recursion (5.5) for a type $\tau$ we make use of the following (conjectural) structure about $C_{\tau}(\delta)$ which has been verified for all types $\tau$ with $\operatorname{def}(\tau) \leq 8$. This refines an observation of Göttsche [8, Remark $4.2(2)]$ about the first 28 (conjectural) coefficients of the node polynomial $N_{\delta}(d)$.

Conjecture 5.5. All entries of $C_{\tau}(\delta)$ are of the form $\frac{3^{\delta}}{\delta !}$ times a polynomial in $\delta$. 
Now, to solve recursion (5.5), we first extend the natural partial order on the types $\tau$ given by $|\tau|=\sum_{j \geq 2} \tau_{j}$ to a linear order with smallest element $\tau=(0,0, \ldots)$. For example, for $N=4$, we could take

$$
(0,0,0)<(1,0,0)<(0,1,0)<(0,0,1)<(1,1,0)<(2,0,0)<(3,0,0) .
$$

Then solve recursion (5.5) for each $\tau$, in increasing order, using the lowertriangularity of the matrices $M_{i}$. For example, to compute the second entry $\frac{3^{\delta}}{\delta !} p(\delta)$ of $C_{1,1}(\delta)$ (assuming Conjecture 5.5), where $p(\delta)$ is a polynomial in $\delta$, we need to solve

$$
C_{1,1}(\delta)=M_{1}(2 \delta-5) C_{1,1}(\delta-1)+M_{2}(2 \delta-6) C_{0,1}(\delta-2)+M_{3}(2 \delta-7) C_{1,0}(\delta-3),
$$

or, explicitly,

$$
\left[\begin{array}{c}
\frac{3}{\delta}^{*} p(\delta) \\
\vdots
\end{array}\right]=\left[\begin{array}{ccc}
* & 0 & 0 \\
* & * & 0 \\
\vdots & \vdots & \ddots
\end{array}\right]\left[\begin{array}{c}
{\frac{3^{\delta-1}}{(\delta-1) !}}^{*}(\delta-1) \\
\vdots
\end{array}\right]+\left[\begin{array}{ccc}
* & 0 & 0 \\
* & * & 0 \\
\vdots & \vdots & \ddots
\end{array}\right]\left[\begin{array}{c}
* \\
* \\
\vdots
\end{array}\right]+\left[\begin{array}{ccc}
* & 0 & 0 \\
* & * & 0 \\
\vdots & \vdots & \ddots
\end{array}\right]\left[\begin{array}{c}
* \\
* \\
\vdots
\end{array}\right] .
$$

The $*$-entries in the vectors $C_{0,1}$ and $C_{1,0}$ are known by a previous computation. The *-entries in $M_{1}, M_{2}$ and $M_{3}$ are given by (5.3). The proof of Lemma 5.1 implies that all denominators of $M_{i}(a)$ in row $j$ are $a+i-j+2$ or 1 (after cancellation). To compute $p(\delta)$, or, more generally, the $j$ th entry in $C_{\tau}(\delta)$, we first clear all denominators and then solve the polynomial difference equation with initial conditions

$$
\begin{aligned}
(2 \delta-\operatorname{def}(\tau)-j+1) 3 p(\delta) & =p(\delta-1)+q(\delta), \\
p\left(\delta_{0}(\tau)\right) & =C_{\tau}\left(\delta_{0}(\tau)\right),
\end{aligned}
$$

Data: A positive integer $N$.

Result: The coefficient vector $C$ of the first $N$ coefficients of $N_{\delta}(d)$.

begin

Compute all templates $\Gamma$ with $\delta(\Gamma) \leq N$;

forall the types $\tau$ with $\operatorname{def}(\tau)<N$ do

Compute initial values $C_{\tau}\left(\delta_{0}(\tau)\right)$ using (5.3), with $\delta_{0}(\tau)$ as in (5.6);

Solve recursion (5.5) for first $N-\operatorname{def}(\tau)$ coordinates of $C_{\tau}(\delta)$;

Set

$$
\begin{aligned}
C_{\tau}^{\mathrm{end}}(\delta) \leftarrow & \sum_{i: \tau_{i} \neq 0} M_{i}^{\mathrm{end}}(2 \delta-i-1-\operatorname{def}(\tau)) C_{\tau \downarrow i}(\delta-i) \\
& +M_{1}^{\mathrm{end}}(2 \delta-2-\operatorname{def}(\tau)) C_{\tau}(\delta-1) ;
\end{aligned}
$$

end

$C \leftarrow 0$

forall the types $\tau$ with $\operatorname{def}(\tau)<N$ do

Shift the entries of $C_{\tau}^{\text {end }}(\delta)$ down by $\operatorname{def}(\tau)$;

$C \leftarrow C+$ shifted $C_{\tau}^{\text {end }}(\delta)$;

end

end

Algorithm 3: Computation of the leading coefficients of the node polynomial. 
where $q(\delta)$ is a rather complicated polynomial depending on earlier calculations and $\delta_{0}(\tau)$ is as in (5.6). One way to solve (5.7) is to bound the degree of the polynomial $p(\delta)$ and solve the corresponding linear system.

Note that a difference equation of the form (5.7) need not have a polynomial solution in general. Conjecture 5.5 is equivalent to all recursions (5.7) appearing in Algorithm 3 to have a polynomial solution.

As in Section 3 (Remark 3.8), Algorithm 3 can be improved significantly by summing the template polynomials $P(\Gamma, k)$ for templates $\Gamma$ with fixed $\left(k_{\min }(\Gamma), l(\Gamma), \varepsilon(\Gamma)\right)$ in advance. Algorithm 3 has been implemented in Maple. Once the templates are known the bottleneck of the algorithm is the initial value computation which, with an improved implementation, should be faster than the template enumeration. Hence we expect Algorithm 3 to compute the first 14 terms of $N_{\delta}(d)$ in reasonable time.

\section{Appendix A. Node polynomials for $\delta \leq 14$}

An explicit list of $N_{\delta}(d)$, for $\delta \leq 14$, is as below. These polynomials are given implicitly in Theorem 3.1. For $\delta \leq 8$ this agrees with [9, Theorem 3.1]. For $\delta \leq 14$ this coincides with the conjectural (implicit) formulas of [8, Remark 2.5].

$$
\begin{aligned}
& N_{0}(d)=1 \text {, } \\
& N_{1}(d)=3(d-1)^{2} \text {, } \\
& N_{2}(d)=\frac{3}{2}(d-1)(d-2)\left(3 d^{2}-3 d-11\right), \\
& N_{3}(d)=\frac{9}{2} d^{6}-27 d^{5}+\frac{9}{2} d^{4}+\frac{423}{2} d^{3}-229 d^{2}-\frac{829}{2} d+525, \\
& N_{4}(d)=\frac{27}{8} d^{8}-27 d^{7}+\frac{1809}{4} d^{5}-642 d^{4}-2529 d^{3}+\frac{37881}{8} d^{2}+\frac{18057}{4} d-8865, \\
& N_{5}(d)=\frac{81}{40} d^{10}-\frac{81}{4} d^{9}-\frac{27}{8} d^{8}+\frac{2349}{4} d^{7}-1044 d^{6}-\frac{127071}{20} d^{5}+\frac{128859}{8} d^{4} \\
& +\frac{59097}{2} d^{3}-\frac{3528381}{40} d^{2}-\frac{946929}{20} d+153513 \\
& N_{6}(d)=\frac{81}{80} d^{12}-\frac{243}{20} d^{11}-\frac{81}{20} d^{10}+\frac{8667}{16} d^{9}-\frac{9297}{8} d^{8}-\frac{47727}{5} d^{7}+\frac{2458629}{80} d^{6} \\
& +\frac{3243249}{40} d^{5}-\frac{6577679}{20} d^{4}-\frac{25387481}{80} d^{3}+\frac{6352577}{4} d^{2} \\
& +\frac{8290623}{20} d-2699706 \\
& N_{7}(d)=\frac{243}{560} d^{14}-\frac{243}{40} d^{13}-\frac{243}{80} d^{12}+\frac{30861}{80} d^{11}-\frac{38853}{40} d^{10}-\frac{802143}{80} d^{9} \\
& +\frac{3140127}{80} d^{8}+\frac{18650493}{140} d^{7}-\frac{54903831}{80} d^{6}-\frac{72723369}{80} d^{5}+\frac{124680069}{20} d^{4} \\
& +\frac{213537633}{80} d^{3}-\frac{3949576431}{140} d^{2}-\frac{188754021}{140} d+48016791, \\
& N_{8}(d)=\frac{729}{4480} d^{16}-\frac{729}{280} d^{15}-\frac{243}{140} d^{14}+\frac{35721}{160} d^{13}-\frac{25839}{40} d^{12}-\frac{320841}{40} d^{11} \\
& +\frac{11847087}{320} d^{10}+\frac{170823033}{1120} d^{9}-\frac{6685218}{7} d^{8}-\frac{1758652263}{1120} d^{7}
\end{aligned}
$$




$$
\begin{aligned}
& +\frac{1102682031}{80} d^{6}+\frac{59797545}{8} d^{5}-\frac{510928080111}{4480} d^{4}-\frac{3283674393}{1120} d^{3} \\
& +\frac{558215113803}{1120} d^{2}-\frac{3722027733}{56} d-861732459 \text {, } \\
& N_{9}(d)=\frac{243}{4480} d^{18}-\frac{2187}{2240} d^{17}-\frac{729}{896} d^{16}+\frac{121743}{1120} d^{15}-\frac{99549}{280} d^{14}-\frac{824823}{160} d^{13} \\
& +\frac{8776593}{320} d^{12}+\frac{74122857}{560} d^{11}-\frac{2188424421}{2240} d^{10}-\frac{132610923}{70} d^{9} \\
& +\frac{11404136871}{560} d^{8}+\frac{2852923401}{224} d^{7}-\frac{3523392270287}{13440} d^{6}+\frac{4109675615}{448} d^{5} \\
& +\frac{261844582229}{128} d^{4}-\frac{2156232149611}{3360} d^{3}-\frac{29528525065861}{3360} d^{2} \\
& +\frac{438722045999}{168} d+15580950065 \\
& N_{10}(d)=\frac{729}{44800} d^{20}-\frac{729}{2240} d^{19}-\frac{729}{2240} d^{18}+\frac{408969}{8960} d^{17}-\frac{746253}{4480} d^{16}-\frac{1932579}{700} d^{15} \\
& +\frac{10649961}{640} d^{14}+\frac{205722099}{2240} d^{13}-\frac{4375229931}{5600} d^{12}-\frac{38815692777}{22400} d^{11} \\
& +\frac{30958937073}{1400} d^{10}+\frac{3413568339}{224} d^{9}-\frac{3624162885799}{8960} d^{8}+\frac{134470136581}{2800} d^{7} \\
& +\frac{27023302169081}{5600} d^{6}-\frac{22514488581251}{8960} d^{5}-\frac{811909836973903}{22400} d^{4} \\
& +\frac{253124357071961}{11200} d^{3}+\frac{867510616107447}{5600} d^{2} \\
& -\frac{2800250331071}{40} d-283516631436 \\
& N_{11}(d)=\frac{2187}{492800} d^{22}-\frac{2187}{22400} d^{21}-\frac{729}{6400} d^{20}+\frac{150903}{8960} d^{19}-\frac{303993}{4480} d^{18}-\frac{56670273}{44800} d^{17} \\
& +\frac{47717667}{5600} d^{16}+\frac{295979589}{5600} d^{15}-\frac{11410430877}{22400} d^{14}-\frac{4051907631}{3200} d^{13} \\
& +\frac{52491198663}{2800} d^{12}+\frac{3418059518271}{246400} d^{11}-\frac{20587006282467}{44800} d^{10} \\
& +\frac{2236636275459}{22400} d^{9}+\frac{49175916627959}{6400} d^{8}-\frac{1464110674563}{256} d^{7} \\
& -\frac{1946239824069277}{22400} d^{6}+\frac{3767687640687823}{44800} d^{5}+\frac{14264414890838423}{22400} d^{4} \\
& -\frac{940418544772283}{1600} d^{3}-\frac{168280746183263029}{61600} d^{2} \\
& +\frac{5073050867636909}{3080} d+5187507215325 \\
& N_{12}(d)=\frac{2187}{1971200} d^{24}-\frac{6561}{246400} d^{23}-\frac{2187}{61600} d^{22}+\frac{496449}{89600} d^{21}-\frac{136809}{5600} d^{20} \\
& -\frac{1618623}{3200} d^{19}+\frac{674946837}{179200} d^{18}+\frac{2321658693}{89600} d^{17}-\frac{893195181}{3200} d^{16} \\
& -\frac{34334301951}{44800} d^{15}+\frac{289702847403}{22400} d^{14}+\frac{1245724147341}{123200} d^{13} \\
& -\frac{803786361621603}{1971200} d^{12}+\frac{65497548165237}{492800} d^{11}+\frac{16192295343681}{1792} d^{10}
\end{aligned}
$$




$$
\begin{aligned}
& -\frac{792669234543351}{89600} d^{9}-\frac{9506773589164709}{67200} d^{8}+\frac{6296062244021929}{33600} d^{7} \\
& +\frac{11029935159768347}{7168} d^{6}-\frac{582428855393100577}{268800} d^{5} \\
& -\frac{5477484616918678589}{492800} d^{4}+\frac{10067756533588172119}{739200} d^{3} \\
& +\frac{4454424013895459501}{92400} d^{2}-\frac{111952943233924509}{3080} d-95376705265437, \\
& N_{13}(d)=\frac{6561}{25625600} d^{26}-\frac{6561}{985600} d^{25}-\frac{19683}{1971200} d^{24}+\frac{1620567}{985600} d^{23}-\frac{88209}{11200} d^{22} \\
& -\frac{3212703}{17920} d^{21}+\frac{262066023}{179200} d^{20}+\frac{494726373}{44800} d^{19}-\frac{673360047}{5120} d^{18} \\
& -\frac{35350103511}{89600} d^{17}+\frac{20952637821}{2800} d^{16}+\frac{3013479294723}{492800} d^{15} \\
& -\frac{580214902388013}{1971200} d^{14}+\frac{1666286215401123}{12812800} d^{13}+\frac{16384163286402207}{1971200} d^{12} \\
& -\frac{909876952033137}{89600} d^{11}-\frac{7649416285706767}{44800} d^{10}+\frac{25855007471662161}{89600} d^{9} \\
& +\frac{65085797443981191}{25600} d^{8}-\frac{108443195356282427}{22400} d^{7} \\
& -\frac{52991400162927629917}{1971200} d^{6}+\frac{1976324604711031517}{39424} d^{5} \\
& +\frac{13580753080243105219}{70400} d^{4}-\frac{73274705967431063281}{246400} d^{3} \\
& -\frac{68173290776099374391}{80080} d^{2}+\frac{2813974748454890667}{3640} d+1761130218801033 \text {, } \\
& N_{14}(d)=\frac{19683}{358758400} d^{28}-\frac{19683}{12812800} d^{27}-\frac{6561}{2562560} d^{26}+\frac{1751787}{3942400} d^{25}-\frac{4529277}{1971200} d^{24} \\
& -\frac{562059}{9856} d^{23}+\frac{398785599}{788480} d^{22}+\frac{5214288411}{1254400} d^{21}-\frac{4860008991}{89600} d^{20} \\
& -\frac{63174295089}{358400} d^{19}+\frac{332872084467}{89600} d^{18}+\frac{3103879378581}{985600} d^{17} \\
& -\frac{4913807521304691}{27596800} d^{16}+\frac{899178800016807}{8968960} d^{15}+\frac{279086438050359453}{44844800} d^{14} \\
& -\frac{468967272863997483}{51251200} d^{13}-\frac{318443311640108577}{1971200} d^{12} \\
& +\frac{328351365725506869}{985600} d^{11}+\frac{1120586814080571923}{358400} d^{10} \\
& -\frac{9448861028448843949}{1254400} d^{9}-\frac{30880785216736406143}{689920} d^{8} \\
& +\frac{444525313669622586903}{3942400} d^{7}+\frac{11429038221675466251}{24640} d^{6} \\
& -\frac{269709254062572016617}{246400} d^{5}-\frac{74660630664748878665353}{22422400} d^{4} \\
& +\frac{140531359469510983018159}{22422400} d^{3}+\frac{16863931195154225977601}{1121120} d^{2} \\
& -\frac{64314454486825349085}{4004} d-32644422296329680 \text {. }
\end{aligned}
$$




\section{Appendix B. Small Severi degrees}

Below we list the Severi degrees $N^{d, \delta}$ for $0 \leq \delta \leq 14$ and $1 \leq d \leq 13$, which were obtained by Algorithm 1 (also see Remark 3.10). Together with the node polynomials of Appendix A, this is a full description of all Severi degrees $N^{d, \delta}$ for $\delta \leq 14$, see Theorem 1.3. The solid line segments indicate the polynomial threshold $d^{*}(\delta)$ of $N^{d, \delta}$. The dashed line segments illustrate the threshold of our Theorem 1.3. The Severi degrees $N^{d, \delta}$ in italic agree with the Gromov-Witten invariants $N_{d, \frac{(d-1)(d-2)}{2}-\delta}$, as for $d \geq \delta+2$, every plane degree $d$ curve with $\delta$ nodes is irreducible.
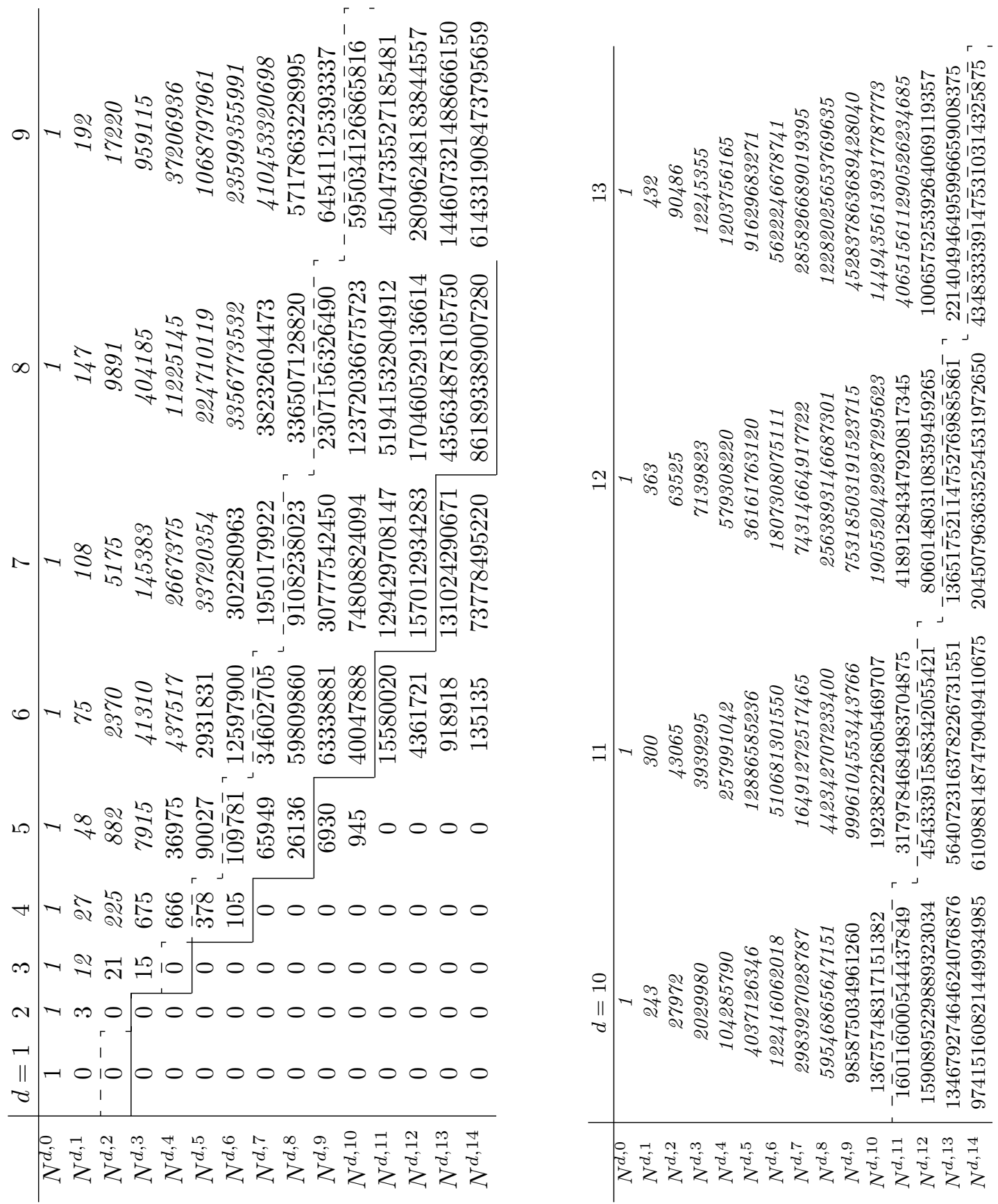


\section{Acknowledgments}

I am thankful to Sergey Fomin for suggesting this problem and fruitful guidance. I also thank the anonymous referee, Erwan Brugallé, Grigory Mikhalkin and Gregg Musiker for valuable comments and suggestions. Part of this work was accomplished at the MSRI (Mathematical Sciences Research Institute) in Berkeley, CA, USA, during the semester program on tropical geometry. I thank MSRI for hospitality.

The author was partially supported by the NSF grant DMS-055588.

\section{References}

[1] F. Block, Relative node polynomials for plane curves, preprint, arXiv:1009.5063, 2010.

[2] F. Block, A. Gathmann and H. Markwig, Psi-floor diagrams and a Caporaso-Harris type recursion, Israel J. Math. (2011), (to appear).

[3] E. Brugallé and G. Mikhalkin, Enumeration of curves via floor diagrams, C. R. Math. Acad. Sci. Paris 345(6) (2007), 329-334.

[4] E. Brugallé and G. Mikhalkin, Floor decompositions of tropical curves: the planar case, in 'Proceedings of Gökova geometry-topology conference 2008', Gökova Geometry/Topology Conference (GGT), Gökova, 2009, 64-90.

[5] L. Caporaso and J. Harris, Counting plane curves of any genus, Invent. Math. 131(2) (1998) 345-392.

[6] P. Di Francesco and C. Itzykson, Quantum intersection rings, in 'The moduli space of curves' (Texel Island, 1994), Progr. Math. 129, Birkhäuser Boston, Boston, MA, 1995, 81-148.

[7] S. Fomin and G. Mikhalkin, Labeled floor diagrams for plane curves, J. Eur. Math. Soc. (JEMS) 12(6) (2010), 1453-1496.

[8] L. Göttsche, A conjectural generating function for numbers of curves on surfaces, Comm. Math. Phys. 196(3) (1998), 523-533.

[9] S. Kleiman and R. Piene, Node polynomials for families: methods and applications, Math. Nachr. 271 (2004), 69-90.

[10] D. Knuth, Johann Faulhaber and sums of powers, Math. Comp. 61(203) (1993), 277-294.

[11] G. Mikhalkin, Enumerative tropical geometry in $\mathbb{R}^{2}$, J. Amer. Math. Soc. 18 (2005), 313-377.

[12] Y.-J. Tzeng, A proof of Göttsche-Yau-Zaslow formula, preprint, arXiv:1009.5371, 2010.

[13] I. Vainsencher, Enumeration of $n$-fold tangent hyperplanes to a surface, J. Algebraic Geom. 4(3) (1995), 503-526.

[14] R. Vakil, Counting curves on rational surfaces, Manuscripta Math. 102(1) (2000), 53-84.

Department of Mathematics, University of Michigan, Ann Arbor, Mi 48109, USA

E-mail address: blockf@umich.edu 
